Pacific Journal of Mathematics

BLOCH FUNCTIONS OF SEVERAL COMPLEX VARIABLES 


\title{
BLOCH FUNCTIONS \\ OF SEVERAL COMPLEX VARIABLES
}

\author{
Xiangyang LiU
}

\begin{abstract}
We extend the concept of Bloch functions and Bloch norm in one complex variable to holomorphic functions defined in the unit ball $\mathbb{B}$ of $\mathbb{C}^{n}$ with values in $\mathbb{C}^{n}$. It is known that in several complex variables the Bloch's Theorem will fail if we do not put some additional restrictions on functions besides the usual normalization of the derivative at the origin. We shall show that many important properties for Bloch functions in one complex variable have analogs for functions in several complex variables. In particular, we generalize Bonk's Distortion Theorem. As applications, we give lower and upper bounds of Bloch constants for various subfamilies of Bloch functions defined in $\mathbb{B}$.
\end{abstract}

1. Introduction. Let $\mathbb{C}$ be the complex plane. It is known that many results in geometric function theory of one complex variable are no longer true in several complex variables. In particular, Bloch's Theorem fails in several complex variables if there is no additional restriction on the class of functions considered (see [2]). K. T. Hahn [3] proved that the conclusion of Bloch's Theorem is true for some families of holomorphic functions defined in the unit ball of $\mathbb{C}^{n}$ with values in $\mathbb{C}^{n}$ (such as the family of bounded holomorphic functions); he also estimated the Bloch constants for these families. R. M. Timoney [7] studied Bloch functions defined in certain domains of $\mathbb{C}^{n}$ with values in $\mathbb{C}$. Sheng Gong [8] also did some work on the Bloch constant in several complex variables.

In this paper, we would like to discuss some properties of Bloch functions defined in the unit ball of $\mathbb{C}^{n}$ with values in $\mathbb{C}^{n}$ (some mathematicians may prefer to use the term "Bloch Mapping" instead of Bloch function). In particular, we shall prove a generalization of Bonk's Distortion Theorem for Bloch functions (see [1] and [4] for Bonk's Distortion Theorem in one complex variable). As applications, we shall give lower bounds of the related Bloch constants. The definition of Bloch functions in this paper is closely related to Timoney's definition.

Let us first introduce some notation. We write a point (or a vector) 
$z \in \mathbb{C}^{n}$ in the following $n \times 1$ matrix form

$$
z=\left(\begin{array}{c}
z_{1} \\
z_{2} \\
\vdots \\
z_{n}
\end{array}\right) .
$$

Consider $\mathbb{C}^{n}$ as an $n$-dimensional Hilbert space with the inner product and the absolute value given by

$$
\langle z, w\rangle=\sum_{i=1}^{n} z_{i} \bar{w}_{i}, \quad|z|=\left(\sum_{i=1}^{n}\left|z_{i}\right|^{2}\right)^{1 / 2},
$$

where $z, w \in \mathbb{C}^{n}$. The unit ball of $\mathbb{C}^{n}$ is the set $\mathbb{B}=\mathbb{B}_{n}=\left\{z \in \mathbb{C}^{n}\right.$ : $|z|<1\}$, and the unit sphere of $\mathbb{C}^{n}$ is denoted by $\partial \mathbb{B}=\left\{z \in \mathbb{C}^{n}\right.$ : $|z|=1\}$.

A function $f: \mathbb{B} \rightarrow \mathbb{C}^{n}$ can also be written in the $n \times 1$ matrix form

$$
f=\left(\begin{array}{c}
f_{1} \\
f_{2} \\
\vdots \\
f_{n}
\end{array}\right),
$$

where each component function $f_{i}$ is a function from $\mathbb{B}$ to $\mathbb{C}$. Denote by $H(\mathbb{B})$ the family of all holomorphic functions from $\mathbb{B}$ to $\mathbb{C}^{n}$. The derivative of a function $f \in H(\mathbb{B})$ at a point $a \in \mathbb{B}$ is the following $n \times n$ matrix (the complex Jacobian of $f$ )

$$
f^{\prime}(a)=\left(\frac{\partial f_{i}}{\partial z_{j}}\right)_{z=a} \text {. }
$$

2. Biholomorphic mappings of $\mathbb{B}$ onto $\mathbb{B}$. With an $n \times n$ matrix $A=\left(a_{i j}\right), a_{i j} \in \mathbb{C}$, we can associate a linear mapping $L_{A}: \mathbb{C}^{n} \rightarrow \mathbb{C}^{n}$ by $L_{A}(z)=A z$, where $A z$ denotes the matrix product of $A$ and $z$. The matrix $A$ (or the linear mapping $L_{A}$ ) is called unitary if

$$
|A z|=|z|
$$

for all $z \in \mathbb{B}$. The determinant of an $n \times n$ matrix $A$ is denoted by $\operatorname{det}(A)$, and the absolute value of $A$ (or the norm of $L_{A}$ ) is given by

$$
|A|\left(=\left|L_{A}\right|\right)=\sup \{|A z|: z \in \mathbb{B}\}=\max \{|A z|: z \in \partial \mathbb{B}\} .
$$

So a unitary matrix always has absolute value 1 . For convenience, we sometimes do not distinguish between a matrix and the associated linear mapping. 
Denote by $\operatorname{Aut}(\mathbb{B})$ the group of all biholomorphic mappings of $\mathbb{B}$ onto $\mathbb{B}$. An element of $\operatorname{Aut}(\mathbb{B})$ is sometimes called a biholomorphic automorphism of $\mathbb{B}$. Obviously, $L_{A} \in \operatorname{Aut}(\mathbb{B})$ if and only if $L_{A}$ is unitary.

For $a \in \mathbb{B}$, define $\varphi_{a}: \mathbb{B} \rightarrow \mathbb{C}^{n}$ by

$$
\varphi_{a}(z)=\frac{a-P_{a} z-\left(1-|a|^{2}\right)^{1 / 2} Q_{a} z}{1-\langle z, a\rangle},
$$

where

$$
P_{a} z=\frac{\langle z, a\rangle}{|a|^{2}} a
$$

is the projection of $\mathbb{C}^{n}$ onto the plane $\left\{z \in \mathbb{C}^{n}: z=u a\right.$ for some $u \in \mathbb{C}\}$ (for $a=0$ we let $P_{a}=0$ ), and $Q_{a}=I-P_{a}$ is the projection of $\mathbb{C}^{n}$ onto the orthogonal complement of the above plane, where $I$ denotes the identity mapping or the identity matrix. The results in the following lemma can be found in Rudin's book [6, pp. 23-30].

Lemma 1. (1) For any $a \in \mathbb{B}, \varphi_{a} \in \operatorname{Aut}(\mathbb{B})$ and $\varphi_{a} \circ \varphi_{a}=I$.

(2) $\varphi_{a}(0)=a, \varphi_{a}(a)=0$, and

$$
\varphi_{a}^{\prime}(0)=-\left(1-|a|^{2}\right) P_{a}-\left(1-|a|^{2}\right)^{1 / 2} Q_{a} .
$$

(3) $\operatorname{Aut}(\mathbb{B})=\left\{L_{A} \circ \varphi_{a}: a \in \mathbb{B}\right.$ and $A$ is a unitary matrix $\}=\left\{\varphi_{b} \circ\right.$ $L_{B}: b \in \mathbb{B}$ and $B$ is a unitary matrix $\}$.

(4) If $\varphi \in \operatorname{Aut}(\mathbb{B})$, then

$$
\left|\operatorname{det} \varphi^{\prime}(z)\right|=\left[\left(1-|\varphi(z)|^{2}\right) /\left(1-|z|^{2}\right)\right]^{(n+1) / 2} .
$$

Lemma 1 will be used repeatedly in the proofs of this paper.

3. Bloch functions in $\mathbb{B}$. The ball in $\mathbb{C}^{n}$ with center $w_{0}$ and radius $r$ is the set

$$
B\left(w_{0}, r\right)=\left\{w \in \mathbb{C}^{n}:\left|w-w_{0}\right|<r\right\} .
$$

For $f \in H(\mathbb{B})$, a schlicht ball of $f$ centered at $f(a)$ is a ball with center $f(a)$ such that $f$ maps an open subset of $\mathbb{B}$ containing $a$ biholomorphically onto this ball. For a point $a \in \mathbb{B}$, let $r(a, f)$ be the radius of the largest schlicht ball of $f$ centered at $f(a)$, and $r(f)=\sup \{r(a, f): a \in \mathbb{B}\}$.

It is known that the condition $\operatorname{det}\left(f^{\prime}\left(z_{0}\right)\right) \neq 0 \quad\left(z_{0} \in \mathbb{B}\right)$ is equivalent to that $f$ is locally schlicht (or locally injective) at the point $z_{0}$.

Definition. A point $z_{0}$ is called a critical point of $f$ if $\operatorname{det}\left(f^{\prime}\left(z_{0}\right)\right)$ $=0$, and $f\left(z_{0}\right)$ is called a critical value of $f$ (in one complex variable, 
such a value is called a branch point). We call a point $w_{0} \in \mathbb{C}^{n}$ a boundary point of $f(\mathbb{B})$ if there is a sequence $\left\{z_{n}\right\}$ in $\mathbb{B}$ such $\left\{z_{n}\right\}$ has no limit point in $\mathbb{B}$ and the sequence $\left\{f\left(z_{n}\right)\right\}$ converges to $w_{0}$. The set of all boundary points of $f(\mathbb{B})$ is denoted by $\partial(f(\mathbb{B}))$. Note that in general $\partial(f(\mathbb{B}))$ is not the topological boundary of $f(\mathbb{B})$ in $\mathbb{C}^{n}$.

LemMA 2. Suppose $f \in H(\mathbb{B}), G$ is an open subset of $\mathbb{B}$, and $a \in G$. If $f$ maps $G$ biholomorphically onto the schlicht ball $B(f(a), r(a, f))$, then either $G$ and $\mathbb{B}$ have a common boundary point or there is a critical value $f\left(z_{0}\right)$ on the boundary of the ball $B(f(a), r(a, f))$ with the critical point $z_{0}$ on the boundary of $G$. Therefore, $r(a, f)$ equals the euclidean distance from $f(a)$ to a boundary point of $f(\mathbb{B})$ or to a critical value of $f$.

Proof. Suppose $G$ and $\mathbb{B}$ have no common boundary point. Then there exists a family $\left\{G_{n}\right\}$ of open subsets of $\mathbb{B}$, such that $G_{n} \supset \bar{G}$, $G_{n} \supset \bar{G}_{n+1}$, and

$$
\bigcap\left\{G_{n}: n=1,2, \ldots\right\}=\bar{G} \text {. }
$$

By definition of $B(f(a), r(a, f))$, we can find $z_{n}$ and $z_{n}^{\prime}$ in $G_{n}$ such that $z_{n} \neq z_{n}^{\prime}$ and $f\left(z_{n}\right)=f\left(z_{n}^{\prime}\right)$. Without loss of generality, we may assume that $\left\{z_{n}\right\}$ converges to $z \in \partial G$ and $\left\{z_{n}^{\prime}\right\}$ converges to $z^{\prime} \in \partial G$; here $\partial G$ denotes the topological boundary of $G$. By continuity, $f(z)$ and $f\left(z^{\prime}\right)$ lie on the boundary of $B(f(a), r(a, f))$, and $f(z)=f\left(z^{\prime}\right)$.

If $z=z^{\prime}$, then it is easy to see that $f(z)$ is a critical value of $f$ with the critical point $z$ on the boundary of $G$.

Suppose $z \neq z^{\prime}$. If both $z$ and $z^{\prime}$ are not critical points of $f$, then the equality $f(z)=f\left(z^{\prime}\right)$ and the local injectivity of $f$ at the two points $z$ and $z^{\prime}$ will imply that $f$ is not injective (schlicht) in $G$. This is impossible.

EXAMPLE (Duren and Rudin). For $n=2$ and $\delta>0$, set

$$
f_{\delta}\left(z_{1}, z_{2}\right)=\left(\begin{array}{c}
z_{1} \\
z_{2}+\left(\frac{z_{1}}{\delta}\right)^{2}
\end{array}\right) \text {, }
$$

then $f_{\delta} \in H(\mathbb{B})$, and $f_{\delta}^{\prime}(0)=I$. P. Duren and $\mathrm{W}$. Rudin proved that the image of $f$ does not contain any ball of radius $\delta$, so $r\left(f_{\delta}\right) \leq \delta$ (see [2] for details).

Recall that the Bloch constant in one complex variable is defined by

$$
B=\inf \left\{r(f): f \in H(\mathbb{D}) \text { and } f^{\prime}(0)=1\right\},
$$


where $\mathbb{D}$ is the unit disk in the complex plane $\mathbb{C}$. The above example shows that for $n \geq 2$, $\inf \left\{r(f): f \in H(\mathbb{B})\right.$, and $\left.f^{\prime}(0)=I\right\}=0$. Hence we should not define the Bloch constant in several complex variables to be $\inf \left\{r(f): f \in H(\mathbb{B})\right.$ and $\left.f^{\prime}(0)=I\right\}$. What we will do is place some reasonable restrictions on functions in $H(\mathbb{B})$ so that the restricted family will have a positive Bloch constant.

Definition. A function $f \in H(\mathbb{B})$ is called a Bloch function if the family

$$
\mathscr{F}_{f}=\{g: g(z)=f(\varphi(z))-f(\varphi(0)) \text { for some } \varphi \in \operatorname{Aut}(\mathbb{B})\}
$$

is a normal family.

Hence a holomorphic function $f$ in $\mathbb{B}$ is a Bloch function if and only if each of its component functions is a Bloch function according to Timoney's definition [7]. Recall that a normal family is a family of holomorphic functions defined in a certain domain such that any sequence of functions in this family has a subsequence which converges uniformly on compact subsets of this domain. It is not difficult to see that in the case $n=1$, the above definition is the usual definition for Bloch functions. The following theorem shows that this definition preserves some important properties of Bloch functions of one complex variable.

TheOREM 1. Suppose $f \in H(\mathbb{B})$. Then

(1) $f$ is a Bloch function implies that the quantity

$$
\|f\|=\sup \left\{\left|(f \circ \varphi)^{\prime}(0)\right|: \varphi \in \operatorname{Aut}(\mathbb{B})\right\}
$$

is finite.

(2) $\|f\|$ is finite implies that $f$ is a Bloch function and

$$
\left|f^{\prime}(z)\right| \leq\|f\| /\left(1-|z|^{2}\right)
$$

for all $z \in \mathbb{B}$.

(3) If $0<K<\infty$ and $\left|f^{\prime}(z)\right| \leq K /\left(1-|z|^{2}\right)^{1 / 2}$, then $f$ is a Bloch function and

$$
\|f\| \leq K
$$

(4) $\|f \circ \varphi\|=\|f\|$ for all $\varphi \in \operatorname{Aut}(\mathbb{B})$.

Proof. (1). This is obvious because $\mathscr{F}_{f}$ is a normal family and any function $g \in \mathscr{F}_{f}$ satisfies $g(0)=0$ imply that the set

$$
\left\{\left|(f \circ \varphi)^{\prime}(0)\right|: \varphi \in \operatorname{Aut}(\mathbb{B})\right\}=\left\{\left|g^{\prime}(0)\right|: g \in \mathscr{F}_{f}\right\}
$$

is bounded. 
(2) Let $z \in \mathbb{B}$. By Lemma 1 , we have $\left|\left(\varphi_{z}^{\prime}(0)\right)^{-1}\right| \leq 1 /\left(1-|z|^{2}\right)^{-1}$. For any $\varphi \in \operatorname{Aut}(\mathbb{B})$,

$$
\left|(f \circ \varphi)^{\prime}(z)\right|=\left|\left[\left(f \circ\left(\varphi \circ \varphi_{z}\right)\right)^{\prime}(0)\right]\left(\varphi_{z}^{\prime}(0)\right)^{-1}\right| \leq\|f\| /\left(1-|z|^{2}\right) .
$$

Thus, $\left\{\left|(f \circ \varphi)^{\prime}(z)\right|: \varphi \in \operatorname{Aut}(\mathbb{B})\right\}$ is locally uniformly bounded which implies that $\mathscr{F}_{f}$ is a normal family. By setting $\varphi=I$ in the above inequality we obtain

$$
\left|f^{\prime}(z)\right| \leq\|f\| /\left(1-|z|^{2}\right),
$$

so (2) is proved.

(3) Let $\varphi \in \operatorname{Aut}(\mathbb{B})$. From Lemma $1, \varphi=L \circ \varphi_{a}$ for some $a \in \mathbb{B}$, where $L$ is a unitary linear mapping, and

$$
\left|\varphi^{\prime}(0)\right|=\left|\varphi_{a}^{\prime}(0)\right| \leq\left(1-|a|^{2}\right)^{1 / 2}=\left(1-|\varphi(0)|^{2}\right)^{1 / 2} .
$$

Therefore,

$$
\left|(f \circ \varphi)^{\prime}(0)\right| \leq\left|f^{\prime}(\varphi(0))\right|\left|\varphi^{\prime}(0)\right| \leq\left|f^{\prime}(\varphi(0))\right|\left(1-|\varphi(0)|^{2}\right)^{1 / 2} \leq K<\infty .
$$

Hence $f$ is a Bloch function with $\|f\| \leq K$.

(4) This follows from the fact that $\operatorname{Aut}(\mathbb{B})$ is a group.

REMARK. Theorem 1 shows that $f$ is a Bloch function if and only if $\|f\|<\infty$. We define the quantity $\|f\|$ to be the Bloch norm of $f$. We should point out that the Bloch norm is only a semi-norm in the linear algebra sense.

Now we want to establish a relation between this Bloch norm we just defined and the Bloch norm in [7] defined by using the Bergman metric on $\mathbb{B}$. Let $Q_{f}(z)(z \in \mathbb{B})$ be given by

$$
Q_{f}(z)=\sup \left\{\left|f^{\prime}(z) x\right|: x \in \mathbb{C}^{n} \text { and } H_{z}(x, x)=1\right\} \text {, }
$$

where $H_{z}$ is the Bergman metric on $\mathbb{B}$, that is,

$$
H_{z}(u, v)=\frac{(n+1)\left(\left(1-|z|^{2}\right)\langle u, v\rangle+\langle u, z\rangle\langle z, v\rangle\right)}{2\left(1-|z|^{2}\right)^{2}}
$$

for all $u, v \in \mathbb{C}^{n}$.

Theorem 2. For any $z \in \mathbb{B}$,

$$
\left|(f \circ \varphi)^{\prime}(0)\right|=\sqrt{(n+1) / 2} Q_{f}(z)
$$

for all $\varphi \in \operatorname{Aut}(\mathbb{B})$ satisfying $\varphi(0)=z$. Therefore,

$$
\|f\|=\sqrt{(n+1) / 2} \sup \left\{Q_{f}(z): z \in \mathbb{B}\right\} .
$$


Proof. Suppose $\varphi \in \operatorname{Aut}(\mathbb{B})$ and $z=\varphi(0)$. By Lemma $1, \varphi=$ $\varphi_{z} \circ L$, where $L$ is a unitary linear mapping.

If $u \in \partial \mathbb{B}$, then $v=L u \in \partial \mathbb{B}$. Write $v$ in the form $v=t z+w$, where $t \in \mathbb{C}, w \in \mathbb{C}^{n}$, and $\langle z, w\rangle=0$. Let $x=\varphi_{z}^{\prime}(0) v=\varphi^{\prime}(0) u$. By Lemma 1 (2),

$$
\varphi_{z}^{\prime}(0) z=-\left(1-|z|^{2}\right) z, \quad \varphi_{z}^{\prime}(0) w=-\left(1-|z|^{2}\right)^{1 / 2} w .
$$

By calculation,

$$
x=-\left(1-|z|^{2}\right) t z-\left(1-|z|^{2}\right)^{1 / 2} w,
$$

and

$$
(f \circ \varphi)^{\prime}(0) u=f^{\prime}(z) x, \quad H_{z}(x, x)=(n+1) / 2 .
$$

Conversely, for any $x \in \mathbb{C}^{n}$ with $H_{z}(x, x)=(n+1) / 2$, we can verify that

$$
u=\left(\varphi^{\prime}(0)\right)^{-1} x \in \partial \mathbb{B} .
$$

Hence

$$
\begin{aligned}
\left|(f \circ \varphi)^{\prime}(0)\right| & =\sup \left\{\left|(f \circ \varphi)^{\prime}(0) u\right|:|u|=1\right\} \\
& =\sup \left\{\left|f^{\prime}(z) x\right|: H_{z}(x, x)=(n+1) / 2\right\} \\
& =\sqrt{(n+1) / 2} Q_{f}(z) .
\end{aligned}
$$

The quantity $\sup \left\{Q_{f}(z): z \in \mathbb{B}\right\}$ is the Bloch norm defined in [7] except that the gradient of $f$ has been replaced by $f^{\prime}(z)$ in the definition of $Q_{f}(z)$. By using Theorem 2 we can calculate (or estimate) the Bloch norm of a holomorphic function in $H(\mathbb{B})$ explicitly.

It is easy to see that when $n=1,\|f\|=\sup \left\{Q_{f}(z): z \in \mathbb{B}\right\}$ reduces to the usual Bloch norm. Recall that a holomorphic function $f$ defined in the unit disk $\mathbb{D}$ of the complex plane is a Bloch function if and only if

$$
\sup \left\{\left(1-|z|^{2}\right)\left|f^{\prime}(z)\right|: z \in \mathbb{D}\right\}<\infty .
$$

One interesting question is: Can we use the above inequality to define Bloch functions when $n \geq 2$ ? The next theorem will show that the answer is affirmative.

Lemma 3. (1) Suppose $A=\left(a_{i j}\right), a_{i j} \in \mathbb{C}$, is an $n \times n$ matrix. If there is a point $x \in \mathbb{C}^{n}$ and a constant $K>0$, such that

$$
|A x| \leq K|x| \text { and }|A y| \leq K|y|
$$

for all $y \in \mathbb{C}^{n}$ satisfying $\langle x, y\rangle=0$, then $|A| \leq \sqrt{2} K$. 
(2) Suppose $f \in H(\mathbb{B})$. Let

$$
m(f)=\sup \left\{\left(1-|z|^{2}\right)\left|f^{\prime}(z)\right|: z \in \mathbb{B}\right\} .
$$

Then there is an absolute constant $c \geq 1$, such that for any $z \in \mathbb{B}$,

$$
\left|f^{\prime}(z) z\right| \leq m(f)|z| /\left(1-|z|^{2}\right),
$$

and

$$
\left|f^{\prime}(z) w\right| \leq c m(f)|w| /\left(1-|z|^{2}\right)^{1 / 2}
$$

for any $w \in \mathbb{C}^{n}$ satisfying $\langle z, w\rangle=0$.

Proof. (1) For any $\zeta \in \mathbb{C}^{n}$, we can write $\zeta$ as

$$
\zeta=u x+y,
$$

where $u \in \mathbb{C}, y \in \mathbb{C}^{n}$, and $\langle x, y\rangle=0$. Hence,

$$
|A \zeta|=|u A x+A y| \leq|u||A x|+|A y| \leq K(|u||x|+|y|) \leq \sqrt{2} K|\zeta|,
$$

therefore $|A| \leq \sqrt{2} K$.

(2) The proof of (2) is similar to the proofs of Theorem 4.7 and Lemma 4.8 in Timoney's paper [7]. The inequality

$$
\left|f^{\prime}(z) z\right| \leq m(f)|z| /\left(1-|z|^{2}\right)
$$

follows from the definition of $m(f)$. We need only to show that there is an absolute constant $c \geq 1$ such that

$$
\left|f^{\prime}(z) w\right| \leq c m(f) /\left(1-|z|^{2}\right)^{1 / 2}
$$

for all $w \in \mathbb{C}^{n}$ satisfying $\langle z, w\rangle=0$ and $|w|=1$.

Define $g: \mathbb{C}^{2} \rightarrow \mathbb{C}^{n}$ by

$$
g(u, v)=f(u z+v w) .
$$

Then

$$
\frac{\partial g}{\partial u}(u, v)=f^{\prime}(u z+v w) z
$$

By Cauchy's Integral Formula,

$$
\frac{\partial^{2} g}{\partial u \partial v}(u, 0)=\frac{1}{2 \pi i} \int_{|v|=\alpha} \frac{f^{\prime}(u z+v w) z}{v^{2}} d v \text {. }
$$

By setting

$$
\alpha=\frac{1}{\sqrt{3}}\left(1-|u z|^{2}\right)^{1 / 2}
$$

in the above equality, we can get

$$
\left|\frac{\partial^{2} g}{\partial u \partial v}(u, 0)\right| \leq \frac{3 \sqrt{3}|z| m(f)}{2\left(1-|u z|^{2}\right)^{3 / 2}} .
$$


Here we have used the fact

$$
\begin{aligned}
& \left(1-|u z|^{2}-|v|^{2}\right)\left|f^{\prime}(u z+v w) z\right| \\
& \quad=\left(1-|u z+v w|^{2}\right)\left|f^{\prime}(u z+v w) z\right| \leq m(f)|z| .
\end{aligned}
$$

By calculation,

$$
\begin{gathered}
\left|\frac{\partial g}{\partial v}(1,0)-\frac{\partial g}{\partial v}(0,0)\right|=\left|\int_{0}^{1} \frac{\partial^{2} g}{\partial u \partial v}(t, 0) d t\right| \\
\quad \leq \int_{0}^{1} \frac{3 \sqrt{3}|z| m(f)}{2\left(1-|t z|^{2}\right)^{3 / 2}} d t=\frac{3 \sqrt{3}|z| m(f)}{2\left(1-|z|^{2}\right)^{1 / 2}}
\end{gathered}
$$

Therefore,

$$
\begin{aligned}
\left|f^{\prime}(z) w\right| & =\left|\frac{\partial g}{\partial v}(1,0)\right| \leq \frac{3 \sqrt{3}|z| m(f)}{2\left(1-|z|^{2}\right)^{1 / 2}}+\left|\frac{\partial g}{\partial v}(0,0)\right| \\
& =\frac{3 \sqrt{3}|z| m(f)}{2\left(1-|z|^{2}\right)^{1 / 2}}+\left|f^{\prime}(0) w\right| \\
& \leq\left(\frac{3 \sqrt{3}|z|}{2\left(1-|z|^{2}\right)^{1 / 2}}+1\right) m(f) \leq \frac{\sqrt{31}}{2} m(f) /\left(1-|z|^{2}\right)^{1 / 2} .
\end{aligned}
$$

Hence we can choose $c=\sqrt{31} / 2$ and (2) is proved.

THEOREM 3. There is an absolute constant $C>0$ such that

$$
m(f) \leq\|f\| \leq C m(f)
$$

for all $f \in H(\mathbb{B})$. Therefore, $f$ is a Bloch function if and only if

$$
m(f)=\sup \left\{\left(1-|z|^{2}\right)\left|f^{\prime}(z)\right|: z \in \mathbb{B}\right\}<\infty .
$$

Proof. The inequality $m(f) \leq\|f\|$ follows from Theorem $1(2)$.

Given $\varphi \in \operatorname{Aut}(\mathbb{B})$, by Lemma $1, \varphi=\varphi_{z} \circ L$, where $z \in \mathbb{B}$ and $L$ is a unitary linear mapping. Further, $\varphi(0)=\varphi_{z}(0)=z$ and $\varphi_{z}^{\prime}(0)=$ $-\left(1-|z|^{2}\right) P_{z}-\left(1-|z|^{2}\right)^{1 / 2} Q_{z}$.

Let $x=L^{-1}(z)$. Then by Lemma 3(2),

$$
\begin{aligned}
\left|(f \circ \varphi)^{\prime}(0) x\right| & =\left|f^{\prime}(z)\left(\varphi_{z}^{\prime}(0) z\right)\right| \\
& =\left(1-|z|^{2}\right)\left|f^{\prime}(z) z\right| \leq m(f)|z|=m(f)|x| .
\end{aligned}
$$

For any $y \in \mathbb{C}^{n}$ satisfying $\langle x, y\rangle=0$, let $w=L(y)$, then $\langle z, w\rangle=$ $\langle x, y\rangle=0$. Lemma $3(2)$ shows that there is an absolute constant $c \geq 1$, such that

$$
\begin{aligned}
\left|(f \circ \varphi)^{\prime}(0) y\right| & =\left|f^{\prime}(z)\left(\varphi_{z}^{\prime}(0) w\right)\right| \\
& =\left(1-|z|^{2}\right)^{1 / 2}\left|f^{\prime}(z) w\right| \leq c m(f)|w|=c m(f)|y| .
\end{aligned}
$$


By Lemma 3(1), $\left|(f \circ \varphi)^{\prime}(0)\right| \leq \sqrt{2} c m(f)$, and hence $\|f\| \leq C m(f)$ with $C=\sqrt{2}$. In fact, the proof of Lemma 2 gives $C=\sqrt{2} c=$ $\sqrt{31 / 2}<4$.

4. Bloch constant for functions defined in $\mathbb{B}$. Now we are ready to define the Bloch constant for various subfamilies of Bloch functions in $\mathbb{B}$.

Definition. Let $\mathscr{B}(K)=\{f \in H(\mathbb{B}):\|f\| \leq K\}, 1 \leq K<\infty$. Define

$$
B(K)=\inf \left\{r(f): f \in \mathscr{B}(K) \text { and } \operatorname{det}\left(f^{\prime}(0)\right)=1\right\} .
$$

We call $B(K)$ the Bloch constant of order $K$.

We have added the restriction $\|f\| \leq K$ in the above definition of the Bloch constant. Later we shall see that $B(K)$ is a positive constant which depends on $K$ when $n \geq 2$. Therefore, the Bloch norm \|\| plays an essential role in the estimation of $B(K)$. It turns out that there is another quantity which is also important. This quantity is given by

$$
\begin{aligned}
\|f\|_{0} & =\sup \left\{\left|\operatorname{det}\left(g^{\prime}(0)\right)\right|^{1 / n}: g \in \mathscr{F}_{f}\right\} \\
& =\sup \left\{\left(1-|z|^{2}\right)^{(n+1) /(2 n)}\left|\operatorname{det}\left(f^{\prime}(z)\right)\right|^{1 / n}: z \in \mathbb{B}\right\} .
\end{aligned}
$$

The second equality follows from the identity

$$
\left|\operatorname{det}\left(\varphi^{\prime}(0)\right)\right|=\left(1-|\varphi(0)|^{2}\right)^{(n+1) / 2}, \quad \varphi \in \operatorname{Aut}(\mathbb{B}),
$$

which is a special case of Lemma $1(4)$. We call $\|f\|_{0}$ the prenorm of $f$. I would like to thank the referee for pointing out that the quantity \|\|$_{0}$ does satisfy the triangle inequality.

THEOREM 4. If $f \in H(\mathbb{B})$, then

(1) $\|f\|_{0} \leq\|f\|$, and equality holds when $n=1$.

(2) $\|f \circ \varphi\|_{0}=\|f\|_{0}$ for all $\varphi \in \operatorname{Aut}(\mathbb{B})$.

(3) $\|f\|_{0}=1$ and $\operatorname{det}\left(f^{\prime}(0)\right)=1$ implies that $\left|\operatorname{det}\left(f^{\prime}(z)\right)\right|=1+$ $o(|z|)$.

(4) For the case $n \geq 2$, there is a constant $c=c_{n}>0$ such that $r(f) \leq c\|f\|_{0}$ for all $f \in H(\mathbb{B})$.

Proof. (1) is true because the inequality $|A|^{n} \geq|\operatorname{det}(A)|$ holds for any $n \times n$ matrix $A$.

(2) follows because $\operatorname{Aut}(\mathbb{B})$ is a group.

(3) is an immediate consequence of the inequality

$$
\left|\operatorname{det}\left(f^{\prime}(z)\right)\right| \leq\left(1-|z|^{2}\right)^{-(n+1) / 2} .
$$


To prove (4), let $B(a)$ be the ball centered at $f(a)$ with radius $r(a, f), V_{n}$ be the volume of $\mathbb{B}$, and

$$
d_{n}=\int_{\mathbb{B}}\left(1-|z|^{2}\right)^{-(n+1)} d v(z),
$$

where the integral is taken with respect to volume. Then $0<c=$ $\left(d_{n} / V_{n}\right)^{1 /(2 n)}<\infty$, and

$$
\begin{aligned}
V_{n} r(a, f)^{2 n} & =\text { the volume of } B(a) \\
& \leq \int_{\mathbb{B}}\left|\operatorname{det}\left(f^{\prime}(z)\right)\right|^{2} d v(z) \leq\|f\|_{0}^{2 n} d_{n},
\end{aligned}
$$

so $r(a, f) \leq c\|f\|_{0}$ for all $a \in \mathbb{B}$ and (4) is proved.

REMARK. Theorem 4(4) implies that $\|f\|_{0}=\|f\|=\infty$ when $r(f)=$ $\infty$ and $n \geq 2$. But unlike the case $n=1$, the converse is not true. For example, let $F \in H(\mathbb{B})$ be the function satisfying

$$
F(0)=0, \quad F^{\prime}(z)=\left(\begin{array}{cc}
1 /\left(1-z_{1}\right)^{n+1} & 0 \\
0 & I_{n-1}
\end{array}\right),
$$

where $I_{n-1}$ is the identity matrix of rank $n-1$. Then $\|F\|_{0}=\|F\|=$ $\infty$ and $r(F) \leq 1<\infty$.

The next theorem is a $\mathbb{C}^{n}$ version of Bonk's Distortion Theorem (see [1] and [4]).

TheOREM 5. If $f \in H(\mathbb{B}),\|f\|_{0}=1$, and $\operatorname{det}\left(f^{\prime}(0)\right)=1$, then $\left|\operatorname{det}\left(f^{\prime}(z)\right)\right| \geq \operatorname{Re}\left\{\operatorname{det}\left(f^{\prime}(z)\right)\right\} \geq(1-\sqrt{n+2}|z|) /(1-|z| / \sqrt{n+2})^{n+2}$ for all $z$ satisfying $|z| \leq 2 \sqrt{n+2} /(n+3)$. The above inequality is best possible.

Proof. For any $\zeta \in \partial \mathbb{B}$, define a holomorphic function $g: \mathbb{D} \rightarrow \mathbb{C}$ by

$$
g(u)=\left(1-a_{n} T(u)\right)^{(n+1)} \operatorname{det}\left(f^{\prime}(T(u) \zeta)\right),
$$

where

$$
T(u)=a_{n}(1-u) /\left(1-a_{n}^{2} u\right), \quad a_{n}=1 /(n+2)^{1 / 2} .
$$

Note that $T$ maps $\mathbb{D}$ onto the disk $D=\left\{w:\left|1-a_{n} w\right|^{2}<1-|w|^{2}\right\} \subset$ $\mathbb{D}$. By calculation, $g(1)=1$. From Theorem $4(3), g^{\prime}(1)=1$. Since $\|f\|_{0}=1$ and $T(\mathbb{D})=D$, we have

$$
\begin{aligned}
|g(u)| & =\left|1-a_{n} T(u)\right|^{(n+1)}\left|\operatorname{det}\left(f^{\prime}(T(u) \zeta)\right)\right| \\
& \leq\left(1-|T(u)|^{2}\right)^{(n+1) / 2}\left|\operatorname{det}\left(f^{\prime}(T(u) \zeta)\right)\right| \leq 1
\end{aligned}
$$


for all $u \in \mathbb{D}$. So $g(\mathbb{D}) \subset \mathbb{D}$. The classical Julia Lemma (see [4]) shows that $g$ maps the horodisk

$$
\Delta(r)=\left\{u \in \mathbb{C}:\left(|1-u|^{2} /\left(1-|u|^{2}\right)\right)<r\right\} \quad(r>0)
$$

of $\mathbb{D}$ into itself. In particular,

$$
\operatorname{Re} g(u) \geq u \text { for all } u \in[-1,1],
$$

which is equivalent to

$$
\operatorname{Re}\left\{\operatorname{det}\left(f^{\prime}(v \zeta)\right)\right\} \geq(1-\sqrt{n+2} v) /(1-v / \sqrt{n+2})^{n+2}
$$

for all $v$ satisfying $0 \leq v \leq 2 \sqrt{n+2} /(n+3)$. This inequality is best possible because there is a function $F \in H(\mathbb{B})$ satisfying $F(0)=0$ and

$$
F^{\prime}(z)=\left(\begin{array}{cc}
\frac{1-\sqrt{n+2} z_{1}}{\left(1-z_{1} / \sqrt{n+1}\right)^{n+2}} & 0 \\
0 & I_{n-1}
\end{array}\right) .
$$

It is not difficult to verify that $\|F\|_{0}=1$, and $\operatorname{det}\left(F^{\prime}(0)\right)=1$.

Lemma 4. Suppose $A=\left(a_{i j}\right)$ is an $n \times n$ matrix. If $|A|>0$, then for any unit vector $\zeta \in \partial \mathbb{B}$, the following inequality holds:

$$
|A \zeta| \geq|\operatorname{det}(A)| /|A|^{n-1} \text {. }
$$

Proof. If $A^{*}=\left(\overline{a_{j i}}\right)$, then the product $A^{*} A$ is a positive semidefinite matrix. Let $\lambda_{1}, \lambda_{2}, \ldots, \lambda_{n}\left(0 \leq \lambda_{1} \leq \lambda_{2} \leq \cdots \leq \lambda_{n}\right)$ be the $n$ eigenvalues of the matrix $A^{*} A$. Then

$$
\left(\lambda_{n}\right)^{1 / 2}=\max \{|A v|: v \in \partial \mathbb{B}\}=|A|, \quad\left(\lambda_{1}\right)^{1 / 2}=\min \{|A v|: v \in \partial \mathbb{B}\},
$$

and

$$
|\operatorname{det}(A)|=\left(\lambda_{1} \lambda_{2} \cdots \lambda_{n}\right)^{1 / 2}
$$

Hence,

$$
|A \zeta| \geq\left(\lambda_{1}\right)^{1 / 2} \geq|\operatorname{det}(A)| /|A|^{n-1} .
$$

THEOREM 6. If $f \in \mathscr{B}(K),\|f\|_{0}=1$, and $\operatorname{det}\left(f^{\prime}(0)\right)=1$, then

$$
\begin{aligned}
& r(0, f) \geq C(K, n) \\
& =K^{1-n} \int_{0}^{1 / \sqrt{n+2}}\left[\left(1-t^{2}\right)^{n-1}(1-\sqrt{n+2} t) /(1-t / \sqrt{n+2})^{n+2}\right] d t \\
& \geq K^{1-n} \frac{1}{e} \frac{\sqrt{n+2}}{n}\left(\left(1+\frac{1}{n+1}\right)^{n+1}-2\right) .
\end{aligned}
$$


Proof. We have seen in Lemma 2 that $r(0, f)$ equals either the euclidean distance from $f(0)$ to a boundary point of $f(\mathbb{B})$ or the euclidean distance from $f(0)$ to a critical value of $f$. Hence there exists a line segment $\Gamma$ of euclidean length $r(0, f)$ from $f(0)$ to a point in $\partial(f(\mathbb{B}))$ or to a critical value of $f$. Let $\gamma$ be the inverse image of $\Gamma$ under the mapping $f$. Then by Theorem $5, \gamma$ is a smooth curve from 0 to $\partial \mathbb{B}$ or to a point $z_{0} \in \mathbb{B}$ with $\left|z_{0}\right| \geq 1 / \sqrt{n+2}$ and

$$
\operatorname{det}\left(f^{\prime}\left(z_{0}\right)\right)=0 \text {. }
$$

By Lemma 4,

$$
\begin{aligned}
r(0, f) & =\left|\int_{\Gamma} d w\right|=\int_{\Gamma}|d w|=\int_{\gamma}\left|f^{\prime}(z) d z\right|=\int_{\gamma}\left|f^{\prime}(z) \frac{d z}{|d z|}\right||d z| \\
& \geq \int_{\gamma} \frac{\left|\operatorname{det}\left(f^{\prime}(z)\right)\right|}{\left|f^{\prime}(z)\right|^{n-1}} d|z| \geq \int_{0}^{1 / \sqrt{n+2}} \frac{\mid \operatorname{det}\left(f^{\prime}(z) \mid\right.}{\left|f^{\prime}(z)\right|^{n-1}} d|z| .
\end{aligned}
$$

Theorem 5 and the inequality $\left|f^{\prime}(z)\right| \leq K /\left(1-|z|^{2}\right)$ (see Theorem $1(2))$ imply that the last quantity is not less than $C(K, n)$.

EXAMPLE. Let $F_{K} \in H(\mathbb{B})$ be the function satisfying $F_{K}(0)=0$ and

$$
F_{K}^{\prime}(z)=\left(\begin{array}{cc}
\frac{\left(1-\sqrt{n+2} z_{1}\right)}{K^{n-1}\left(1-z_{1} / \sqrt{n+2}\right)^{n+2}} & 0 \\
0 & K I_{n-1}
\end{array}\right) .
$$

For $K$ sufficiently large, we have

$$
\left|F_{K}^{\prime}(z)\right| \leq K /\left(1-|z|^{2}\right)^{1 / 2},
$$

thus $F_{K} \in \mathscr{B}(K)$ by Theorem $1(3)$. By direct calculations, we obtain

$$
\left\|F_{K}\right\|_{0}=1, \quad \operatorname{det}\left(F_{K}^{\prime}(0)\right)=1,
$$

and

$$
r\left(0, F_{K}\right) \leq e C(K, n) \text {. }
$$

Therefore, the lower bound on $r(0, f)$ in Theorem 6 is a reasonable estimate; it is not off by more than a factor of $e$.

COROLlary. $K^{1-n} \geq B(K) \geq C(K, n)$.

Proof. By definition, there are functions $g_{m} \in \mathscr{B}(K)(m=1,2, \ldots)$ such that

$$
\operatorname{det}\left(g_{m}^{\prime}(0)\right)=1, \quad r\left(g_{m}\right) \rightarrow B(K) .
$$

Note that $1 \leq\left\|g_{m}\right\|_{0} \leq K$. Let $h_{m}=g_{m} /\left\|g_{m}\right\|_{0}$, then

$$
h_{m} \in \mathscr{B}(K), \quad\left\|h_{m}\right\|_{0}=1 \text {. }
$$


A standard normal family argument and Theorem 4(2) imply that

$$
B(K)=\inf \left\{r(f): f \in \mathscr{B}(K) \text { and }\|f\|_{0}=1\right\} .
$$

Therefore,

$$
r\left(g_{m}\right) \geq r\left(h_{m}\right) \rightarrow B(K) .
$$

For each $m$, let

$$
\mathscr{F}_{m}=\left\{f: f(z)=h_{m}(\varphi(z))-h_{m}(\varphi(0)) \text { for some } \varphi \in \operatorname{Aut}(\mathbb{B})\right\} .
$$

By Theorem 1(4) and Theorem 4(2), all functions in $\mathscr{F}_{m}$ have the same prenorm \|\|$_{0}$ and the same Bloch norm \|\| as $h_{m}$ does. Since $h_{m}$ is a Bloch function, $\mathscr{F}_{m}$ is a normal family. Hence we can find a function $f_{m} \in \mathscr{B}(K)$, such that

$$
r\left(f_{m}\right)=r\left(h_{m}\right) \rightarrow B(K), \quad\left\|f_{m}\right\|_{0}=\left\|h_{m}\right\|_{0}=1,
$$

and

$$
\operatorname{det}\left(f_{m}^{\prime}(0)\right)=1
$$

Theorem 6 gives

$$
B(K)=\lim r\left(f_{m}\right) \geq \lim r\left(0, f_{m}\right) \geq C(K, n) .
$$

The upper bound is obtained from the function

$$
F(z)=\left(\begin{array}{c}
K^{1-n} z_{1} \\
K z_{2} \\
\vdots \\
K z_{n}
\end{array}\right) .
$$

REMARK. (1) Note that $C(K, 1)=\sqrt{3} / 4$; this is the well-known lower bound of the classical Bloch constant obtained by L. V. Ahlfors in 1937 (M. Bonk [1] has recently improved the lower bound of the classical Bloch constant to $\left.\sqrt{3} / 4+10^{-14}\right)$.

(2) Let $B$ be the classical Bloch constant (for holomorphic functions of one complex variable). When $K \geq[2 \sqrt{3} / 3]^{1 / n}$, we can improve the upper bound in the above corollary to

$$
K^{1-n} B \geq B(K) .
$$

To demonstrate this, let $H(\mathbb{D})$ be the family of all holomorphic functions from $\mathbb{D}$ to $\mathbb{C}$, then we have the following well-known iden- 
tity which was proved by E. Landau in 1929:

$$
B=\inf \left\{r(f): f \in H(\mathbb{D}),\|f\|=1, \text { and } f^{\prime}(0)=1\right\} .
$$

Note that (since $n=1$ ) in the above identity,

$$
r(f)=\sup \{\text { radii of schlicht disks of } f\},
$$

and $\|f\|$ is the usual Bloch norm of $f$.

Suppose $f \in H(\mathbb{D}),\|f\|=1$, and $f^{\prime}(0)=1$. We need only to show that

$$
K^{1-n} r(f) \geq B(K)
$$

Define $F_{f} \in H(\mathbb{B})$ by

$$
F_{f}(z)=\left(\begin{array}{c}
K^{1-n} f\left(z_{1}\right) \\
K z_{2} \\
\vdots \\
K z_{n}
\end{array}\right) .
$$

It is elementary but lengthy to verify that $\left\|F_{f}\right\|=K$ when $K \geq$ $[2 \sqrt{3} / 3]^{1 / n}$ (see [5] for details). Also, $\operatorname{det}\left(F_{f}^{\prime}(0)\right)=1$. Hence $F_{f} \in$ $\mathscr{B}(K)$, and

$$
K^{1-n} r(f) \geq r\left(F_{f}\right) \geq B(K) .
$$

In fact, if $\left\|F_{f}\right\| \leq K$ is true for all $K \geq 1$, then by the same argument, $B(K) \leq K^{1-n} B$ will be true for all $K \geq 1$.

5. Corresponding results for locally schlicht functions. We know that a function $f \in H(\mathbb{B})$ is locally schlicht in $\mathbb{B}$ if and only if $\operatorname{det}\left(f^{\prime}(z)\right)$ does not vanish in $\mathbb{B}$. Recall that in the geometric function theory of one complex variable, the locally schlicht Bloch constant is defined to be

$$
B_{0}=\inf \left\{r(f): f \in H(\mathbb{D}), f^{\prime}(0)=1, \text { and } f^{\prime}(z) \neq 0 \text { for all } z \in \mathbb{D}\right\} .
$$

Liu and Minda [4] established a sharp distortion theorem (an analog of Bonk's Distortion Theorem) for locally schlicht Bloch functions of one complex variable. For several complex variables, the analogs of Theorem 5 and Theorem 6 (and its corollary) hold. We will omit the proofs because the ideas are the same (see [5] for details).

For $1 \leq K<\infty$, define

$$
\begin{gathered}
\mathscr{B}_{0}(K)=\left\{f \in \mathscr{B}(K): \operatorname{det}\left(f^{\prime}(z)\right) \neq 0 \text { for all } z \in \mathbb{B}\right\}, \\
B_{0}(K)=\inf \left\{r(f): f \in \mathscr{B}_{0}(K), \text { and } \operatorname{det}\left(f^{\prime}(0)\right)=1\right\} .
\end{gathered}
$$


Theorem 7. Suppose $f \in H(\mathbb{B}),\|f\|_{0}=1$, and $\operatorname{det}\left(f^{\prime}(0)\right)=1$. If $\operatorname{det}\left(f^{\prime}(z)\right) \neq 0$ for all $z \in \mathbb{B}$, then

$$
\left|\operatorname{det}\left(f^{\prime}(z)\right)\right| \geq(1-|z|)^{-(n+1)} \exp \{-(n+1)|z| /(1-|z|)\}
$$

for all $z \in \mathbb{B}$. This inequality is best possible.

The following function is an extremal function for Theorem 7.

$$
F(z)=\left(\begin{array}{c}
\frac{1}{\left(1-z_{1}\right)^{n+1}} \exp \left\{-(n+1) z_{1} /\left(1-z_{1}\right)\right\} \\
z_{2} \\
\vdots \\
z_{n}
\end{array}\right) \text {. }
$$

THEOREM 8. If $f \in \mathscr{B}_{0}(K)$ satisfies the conditions of Theorem 7, then $r(0, f) \geq C_{0}(K, n)=K^{1-n} \int_{0}^{1} \frac{\left(1-t^{2}\right)^{n-1}}{(1-t)^{n+1}} \exp \{-(n+1) t /(1-t)\} d t$

COROLlaRy. $K^{1-n} \geq B_{0}(K) \geq C_{0}(K, n)$.

REMARK. (1) $C_{0}(K, 1)=1 / 2$ is currently the largest known lower bound for the locally schlicht Bloch constant of one complex variable.

(2) By the same argument as in $\S 4, B_{0}(K) \leq K^{1-n} B_{0}$ is true when $K \geq[2 \sqrt{3} / 3]^{1 / n}$.

(3) Finally, we would like to point out that all of the results in this paper have analogs for holomorphic functions defined in the unit polydisc of $\mathbb{C}^{n}$, see [5].

Acknowledgment. This paper constitutes part of my Ph.D. dissertation. I would like to thank my thesis advisor, Professor David Minda, for his many valuable suggestions.

\section{REFERENCES}

[1] M. Bonk, On Bloch's constant, Proc. Amer. Math. Soc., 110 (1990), 889-894.

[2] P. Duren and W. Rudin, Distortion in several variables, Complex Variables, 5 (1986), 323-326.2.

[3] K. T. Hahn, Higher diimensional generalizations of the Bloch constant and their lower bounds, Trans. Amer. Math. Soc., 179 (1973), 263-274.

[4] X. Liu and D. Minda, Distortion theorems for Bloch functions, Transl. Amer. Math. Soc., to appear.

[5] X. Liu, Topics in geometric function theory, Ph.D. Dissertation, University of Cincinnati, in preparation.

[6] W. Rudin, Function Theory in the Unit Ball of $\mathbb{C}^{n}$, Springer-Verlag, New York, 1980. 
[7] R. M. Timoney, Bloch functions in several complex variables I, Bull. London Math. Soc., 12 (1980), 241-267.

[8] Sheng Gong, private communication.

Received July 15, 1990 and in revised form December 20,1990. Research partially supported by NSF Grant No. DMS-8801439, a Distinguished Dissertation Assistantship awarded by the University of Cincinnati, and a Student Summer Research Fellowship awarded by the University Research Council of the University of Cincinnati.

UNIVERSITY OF CINCINNATI

CincinNati, OH 45221-0025 



\title{
PACIFIC JOURNAL OF MATHEMATICS EDITORS
}

V. S. VARADARAJAN

(Managing Editor)

University of California

Los Angeles, CA 90024-1555-05

HeRbert Clemens

University of Utah

Salt Lake City, UT 84112

F. Michael Christ

University of California

Los Angeles, CA 90024-1555

THOMAS ENRIGHT

University of California, San Diego

La Jolla, CA 92093
Nicholas ERcolani

University of Arizona

Tucson, AZ 85721

R. FINN

Stanford University

Stanford, CA 94305

VAUGHAN F. R. JONES

University of California

Berkeley, CA 94720

Steven Kerckhoff

Stanford University

Stanford, CA 94305

\section{C. MOORE}

University of California

Berkeley, CA 94720

Martin ScharlemanN

University of California

Santa Barbara, CA 93106

HAROLD STARK

University of California, San Diego

La Jolla, CA 92093

\section{ASSOCIATE EDITORS}
R. Arens
E. F. BECKENBACH
B. H. NeumanN
F. WoLF
K. YoshidA
(1906-1982)
(1904-1989)

\section{SUPPORTING INSTITUTIONS}

UNIVERSITY OF ARIZONA

UNIVERSITY OF BRITISH COLUMBIA

CALIFORNIA INSTITUTE OF TECHNOLOGY

UNIVERSITY OF CALIFORNIA

MONTANA STATE UNIVERSITY

UNIVERSITY OF NEVADA, RENO

NEW MEXICO STATE UNIVERSITY

OREGON STATE UNIVERSITY

\author{
UNIVERSITY OF OREGON \\ UNIVERSITY OF SOUTHERN CALIFORNIA \\ STANFORD UNIVERSITY \\ UNIVERSITY OF HAWAII \\ UNIVERSITY OF TOKYO \\ UNIVERSITY OF UTAH \\ WASHINGTON STATE UNIVERSITY \\ UNIVERSITY OF WASHINGTON
}

The Supporting Institutions listed above contribute to the cost of publication of this Journal, but they are not owners or publishers and have no responsibility for its content or policies.

\begin{abstract}
Mathematical papers intended for publication in the Pacific Journal of Mathematics should be in typed form or offset-reproduced (not dittoed), double spaced with large margins. Please do not use built up fractions in the text of the manuscript. However, you may use them in the displayed equations. Underline Greek letters in red, German in green, and script in blue. The first paragraph must be capable of being used separately as a synopsis of the entire paper. In particular it should contain no bibliographic references. Please propose a heading for the odd numbered pages of less than 35 characters. Manuscripts, in triplicate, may be sent to any one of the editors. Please classify according to the 1991 Mathematics Subject Classification scheme which can be found in the December index volumes of Mathematical Reviews. Supply name and address of author to whom proofs should be sent. All other communications should be addressed to the managing editor, or Elaine Barth, University of California, Los Angeles, California 90024-1555-05.

There are page-charges associated with articles appearing in the Pacific Journal of Mathematics. These charges are expected to be paid by the author's University, Government Agency or Company. If the author or authors do not have access to such Institutional support these charges are waived. Single authors will receive 50 free reprints; joint authors will receive a total of 100 free reprints. Additional copies may be obtained at cost in multiples of 50 .
\end{abstract}

The Pacific Journal of Mathematics (ISSN 0030-8730) is published monthly except for July and August. Regular subscription rate: $\$ 190.00$ a year (10 issues). Special rate: $\$ 95.00$ a year to individual members of supporting institutions.

Subscriptions, orders for numbers issued in the last three calendar years, and changes of address should be sent to Pacific Journal of Mathematics, P.O. Box 969, Carmel Valley, CA 93924, U.S.A. Old back numbers obtainable from Kraus Periodicals Co., Route 100, Millwood, NY 10546.

The Pacific Journal of Mathematics at P.O. Box 969, Carmel Valley, CA 93924 (ISSN 0030-8730) is published monthly except for July and August. Second-class postage paid at Carmel Valley, California 93924, and additional mailing offices. Postmaster: send address changes to Pacific Journal of Mathematics, P.O. Box 969, Carmel Valley, CA 93924.

PUBLISHED BY PACIFIC JOURNAL OF MATHEMATICS, A NON-PROFIT CORPORATION Copyright (C) 1992 by Pacific Journal of Mathematics 


\section{Pacific Journal of Mathematics}

Vol. 152, No. $2 \quad$ February, 1992

Edoardo Ballico, On the restrictions of the tangent bundle of the

Grassmannians

Edward Burger, Homogeneous Diophantine approximation in

$S$-integers

Jan Dijkstra, Jan van Mill and Jerzy Mogilski, The space of

infinite-dimensional compacta and other topological copies of $\left(l_{f}^{2}\right)^{\omega} \ldots 255$

Mike Hoffman, Multiple harmonic series ..................... 275

Wu Hsiung Huang, Superharmonicity of curvatures for surfaces of constant

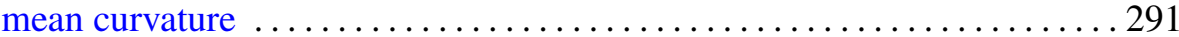

George Kempf, Pulling back bundles ......................... 319



Andrew Solomon Lipson, Some more states models for link invariants . . . . 337

Xiang Yang Liu, Bloch functions of several complex variables .......... 347

Madabusi Santanam Raghunathan, A note on generators for arithmetic

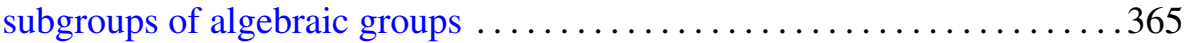

Marko Tadić, Notes on representations of non-Archimedean $\operatorname{SL}(n) \ldots \ldots 375$ 